\title{
Candida albicans and Candida dubliniensis: comparison of biofilm formation in terms of biomass and activity
}

\section{HENRIQUES, J. AZEREDO and R. OLIVEIRA \\ Centro de Engenharia Biológica - CEB, Universidade do Minho, Braga, Portugal}

Accepted: 20 December 2005

\section{Introduction}

Candida albicans is one of the most well-known Candida species, as it is responsible for the majority of infections with such organisms. More recently, another species, C. dubliniensis, which is closely related to C. albicans, was recovered from the oral cavity of human immunodeficiency virus (HIV)-infected patients. ${ }^{1}$ Furthermore, presence of this species in cases of superficial and systemic disease in immunocompromised individuals has been reported. ${ }^{2,3}$

As C. dubliniensis is only rarely recovered from the oral cavity of healthy individuals, its prevalence in HIVinfected and acquired immune deficiency syndrome (AIDS) patients indicates that it is an opportunistic pathogen in such immunocompromised states, probably from the patient's own microbial flora. In this environment, the ability of $C$. dubliniensis to adhere strongly to oral epithelial cells may provide it with a competitive advantage over other, less-adherent non-albicans Candida species. $^{4}$

One of the most important virulence factors of Candida species is their ability to form biofilms, and thus they assume greater significance in the clinical context because they are associated with a significantly enhanced ability to express resistance against most antimicrobial agents. ${ }^{5,6}$ Candidiasis is often an implant-related infection in which adherent microbial populations are found on the surfaces of indwelling devices such as catheters, prosthetic heart valves and joint replacements.?

Biofilm infections can be caused by a single microbial species or by a mixture of bacterial and fungal species. ${ }^{8,9}$ Biofilms are notoriously difficult to eliminate and are a source of many resistant infections. ${ }^{10,11}$ Biofilm formation has been largely studied in relation to the effect of antifungal agents. ${ }^{12-14}$

\section{ABSTRACT}

Candida albicans and C. dubliniensis are two species responsible for oral candidiasis, especially in immunocompromised patients. Microbial infection is preceded by adherence and biofilm formation. Biofilm formation represents the most common form of $C$. albicans in the oral cavity and is considered to be one of the most important virulence factors. In this study, the biofilm formation ability of $C$. dubliniensis was compared with that of $C$. albicans in terms of biomass (quantified using crystal violet) and activity (assessed by formazan salts formation). Both species formed heterogeneous biofilms; however, species and strain variations were seen in the quantification of biomass and activity. There was no correlation between pseudohyphae formation and biofilm formation capability.

KEY WORDS: Biofilms. Candida albicans. Candida dubliniensis.

Although fungal infection-associated biofilm formation is less common than the bacterial variety, the former tends to be more serious. ${ }^{15}$ Therefore, many studies have focused on the C. albicans biofilm, due to its well-recognised virulence, whereas only a few studies of the $C$. dubliniensis biofilms have been reported. ${ }^{16-18}$

In a previous study by this group, strains of $C$. albicans and C. dubliniensis were shown to be equally capable of colonising the oral cavity, and that the presence of saliva enhances candidal colonisation. ${ }^{19}$ The present study aims to evaluate the ability of each species to form a biofilm in the oral environment.

\section{Materials and methods}

Cells were grown in Sabouraud dextrose broth (SDB, Merck), which is the most common medium used to grow Candida species, or in an artificial saliva growth medium.

\section{Sabouraud dextrose medium}

Yeast cells were maintained in Sabouraud dextrose agar (SDA) that was prepared according to the manufacturer's instructions (30 g/L) plus $1.7 \%$ agar (Merck). Sabouraud dextrose broth (SDB, Merck; $30 \mathrm{~g} / \mathrm{L}$ in water) was used to grow the cells in liquid medium. 


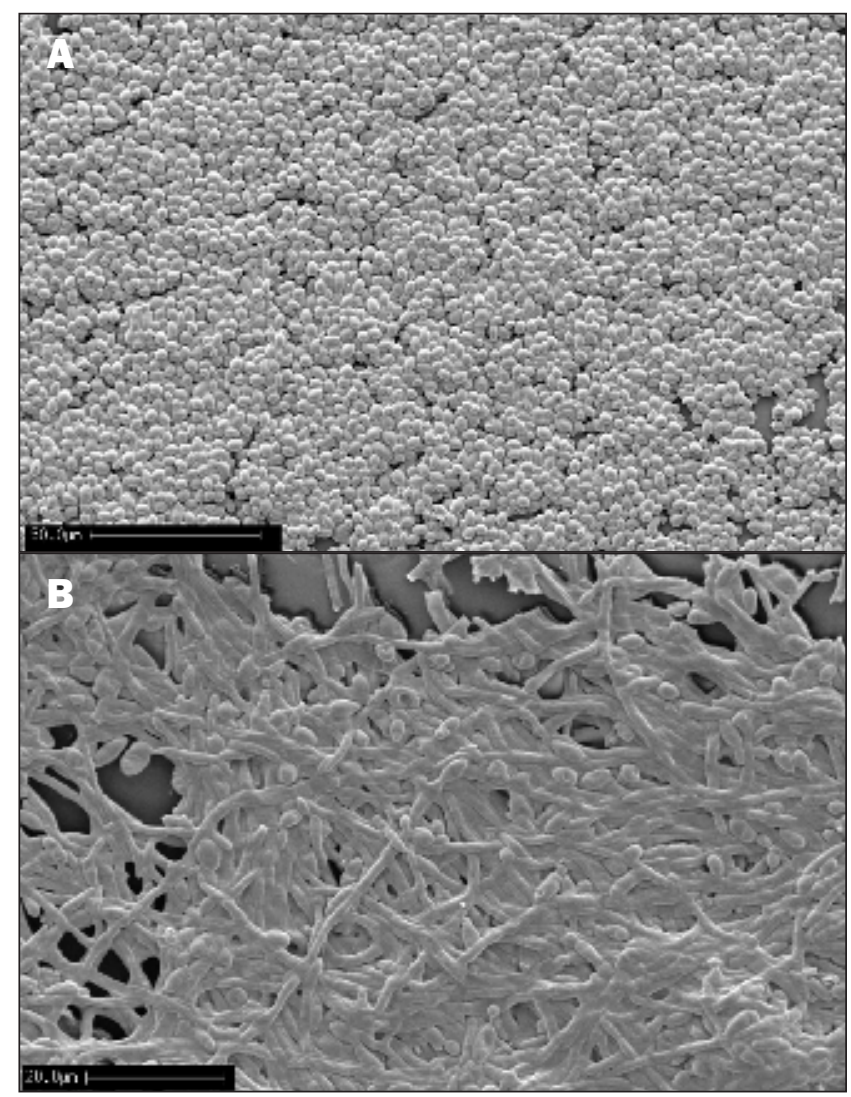

Fig. 1. SEM observation of a general view of a mature biofilm of C. albicans $12 \mathrm{~A}$ at a) $\times 500$ (bar corresponds to $50 \mu \mathrm{m}$ ) and b) $\mathrm{x} 1000$, containing blastopores, hyphae and pseudohyphae (bar corresponds to $20 \mu \mathrm{m}$ ).

\section{Artificial saliva medium}

In some experiments artificial saliva was used to mimic in vivo oral conditions. This was prepared according to Gal et al..$^{20}$ and comprised $125.6 \mathrm{mg} / \mathrm{L} \mathrm{NaCl}, 963.9 \mathrm{mg} / \mathrm{L} \mathrm{KCl}, 189.2 \mathrm{mg} / \mathrm{L} \mathrm{KSCN}$, $654.5 \mathrm{mg} / \mathrm{L} \mathrm{KH}_{2} \mathrm{PO}_{4}, 200 \mathrm{mg} / \mathrm{L}$ Urea, $763.2 \mathrm{mg} / \mathrm{L} \mathrm{Na}_{2} \mathrm{SO}_{4} \cdot 10 \mathrm{H}_{2} \mathrm{O}$, $178 \mathrm{mg} / \mathrm{L} \mathrm{NH} \mathrm{Nl}_{4}, 227.8 \mathrm{mg} / \mathrm{L} \mathrm{CaCl} 2.2 \mathrm{H}_{2} \mathrm{O}$ and $630.8 \mathrm{mg} / \mathrm{L}$ $\mathrm{NaHCO}_{3}$. In order to create an artificial saliva growth medium, glucose $(2 \mathrm{~g} / \mathrm{L})$, yeast extract $(2 \mathrm{~g} / \mathrm{L})$ and peptone $(5 \mathrm{~g} / \mathrm{L})$ were added to the solution. ${ }^{21} \mathrm{pH}$ was adjusted with $\mathrm{CO}_{2}$ to 6.8 .

\section{Yeast cells}

The Candida species used were C. albicans and C. dubliniensis and two different strains of each were assayed. For C. albicans one strain was from American Type Culture Collection, ATCC 32354 (C. albicans B311) and the other was a clinical isolate (C. albicans 12A). For C. dubliniensis two strains were obtained from CBS (C. dubliniensis 7987 and C. dubliniensis 7988). For all assays, yeast cells were grown for $24 \mathrm{~h}$ in SDA at $37^{\circ} \mathrm{C}$. The cells were then inoculated in SDB for $18 \mathrm{~h}$ at $37^{\circ} \mathrm{C}$ and $150 \mathrm{rpm}$. After incubation the cells were harvested by centrifugation for 10 min at $5000 \mathrm{rpm}$ and $4^{\circ} \mathrm{C}$.

\section{Acrylic surfaces}

Acrylic coupons were prepared as described by Samaranayake and MacFarlane. ${ }^{22,23}$ Briefly, $1.5 \mathrm{~g}$ selfpolymerising acrylic powder was mixed with $1 \mathrm{ml}$ monomer in liquid phase. After mixing, the solution was poured onto a surface covered with aluminium foil. After $45 \mathrm{sec}$ another surface was placed on top of the polymerising mixture. After $30 \mathrm{~min}$ the acrylic sheet was cut into squares, $8 \times 8 \mathrm{~mm}^{2}$.

\section{Biofilm formation}

Yeast cells were subcultured in SDA for $24 \mathrm{~h}$ at $37^{\circ} \mathrm{C}$, followed by growth in SDB at $37^{\circ} \mathrm{C}$ and $150 \mathrm{rpm}$ for $18 \mathrm{~h}$. Cells were harvested by centrifugation (5000 rpm, $10 \mathrm{~min}$ ) and resuspended in SDB or in artificial saliva growth medium to $5 \times 10^{7}$ cells $/ \mathrm{mL}$. The biofilm was formed on acrylic coupons 24-well plates, with each well containing $1 \mathrm{~mL}$ yeast cell suspension. The medium (SDB or artificial saliva growth medium) was replaced by fresh medium every $12 \mathrm{~h}$ and biofilm formation was inspected after $7,14,24,48$ and $72 \mathrm{~h}$.

\section{Crystal violet}

Coupons containing biofilm were removed from each well and immersed for $15 \mathrm{~min}$ in wells containing $1 \mathrm{~mL}$ methanol. After withdrawing the methanol, the coupons were allowed to dry at room temperature before adding $600 \mu \mathrm{L}$ crystal violet. After $5 \mathrm{~min}$ the coupons were washed in water and immersed in acetic acid (33\%) to dilute the stain. Absorbance was read at $570 \mathrm{~nm}$.

\section{Tetrazolium salts}

Coupons containing biofilm were withdrawn from each well and immersed in $1 \mathrm{~mL}$ solution of $100 \mu \mathrm{g} / \mu \mathrm{L}$ XTT and 10 $\mu \mathrm{g} / \mu \mathrm{L}$ PMS. The well plate was incubated in the dark for $3 \mathrm{~h}$ with agitation (150 rpm). Each solution was centrifuged for 3 min at $1000 \mathrm{rpm}$ and the absorbance was read at $490 \mathrm{~nm}$.

\section{Statistical analysis}

Data were analysed using the SPSS (Statistical Package for the Social Sciences) program. One-way ANOVA with the Bonferroni test was used to compare the number of adherent cells of the four strains. All tests were performed with a confidence level of $95 \%$.

\section{Results}

Biofilm quantification was performed using two different approaches: total biomass formed using crystal violet (CV) staining; and quantification of the amount of active cells by the reduction of a tetrazolium salt (XTT). Correlation and significance values obtained for the two strains of $C$. albicans and C. dubliniensis are presented in Table 1.

Table 1. Correlation factors $\left(r^{2}\right)$ and significance values $(P)$ obtained for the absorbance of CV-stained suspensions of $C$. albicans B311, C. albicans 12 A, C. dubliniensis 7987 and C. dubliniensis 7988 versus the absorbance of the same cell suspensions stained by XTT

\begin{tabular}{|lcc}
\hline Strain & $r^{2}$ & $P$ \\
\hline Candida albicans B311 & 0.973 & 0.014 \\
\hline Candida albicans 12A & 0.991 & 0.000 \\
\hline Candida dubliniensis 7987 & 0.989 & 0.000 \\
\hline Candida dubliniensis 7988 & 0.978 & 0.001 \\
\hline
\end{tabular}


The values show a statistical significant correlation between the absorbance of both evaluation methods for all the strains studied. Scanning electron microscope (SEM) observations of biofilms formed by C. albicans $12 \mathrm{~A}$ are presented in Figure 1. The images show that the biofilms were thick and heterogeneous, with either the predominance of blastopores or mycelia.

Figures 2 and 3 demonstrate the evolution of the biofilm biomass quantified by crystal violet staining.

In order to aid interpretation of these results the slope between 48 and $72 \mathrm{~h}$ was determined and the values presented in Table 2. The slopes of the biofilm formation profiles for C. albicans B311 and C. dubliniensis 7987 are similar, as is the case with the other two strains.

Biofilm activity profiles formed on either SDB or artificial saliva growth medium are presented in Figures 4 and 5 . The slope of the different curves seen between 48 and $72 \mathrm{~h}$ was determined and is presented in Table 3. Activity of all biofilms formed in SDB in effect levelled after $48 \mathrm{~h}$. The only profile that does not present a null slope corresponds to C. dubliniensis 7987 in artificial saliva.

Table 4 presents the significance values obtained by both quantification methods (CV and XTT). Significant differences were seen among the biofilms formed in both media (95\% confidence level). Comparing the results obtained in terms of biofilm mass and biofilm activity, it can be concluded that cell activity is not dependent on cell number. In the case of a mature biofilm, the number of total cells might be high but their activity can be low. For instances, the cells in the deeper layer may be less active as a result of diffusional limitations.

Presence of hyphae at $48 \mathrm{~h}$ was observed under SEM (Figs. 6 and 7). All the biofilms were compact and it is possible to see some pseudohyphae. C. albicans B311 formed biofilms with more and longer pseudohyphae, both in SDB and in artificial saliva. All other strains formed biofilms that produced pseudohyphae only in artificial saliva growth medium.

\section{Discussion}

Candida spp. biofilms are highly heterogeneous. This mixture of yeast blastopores, hyphae and matrix material is not seen when the organism is grown in liquid culture or on an agar surface, which suggests that morphogenesis is triggered when an organism contacts a surface and that the

Table 2. Values of the slopes of the biofilm mass profiles (between 48 and 72 h) of C. albicans B311, C. albicans 12A, C. dubliniensis 7987 and C. dubliniensis 7988 evaluated with CV in both biofilm formation media

\begin{tabular}{|lcc|}
\hline Strain & \multicolumn{2}{c|}{$\begin{array}{c}\text { Slope } \\
\text { Artificial saliva growth } \\
\text { medium }\end{array}$} \\
\hline Candida albicans B311 & 0.015 & 0.023 \\
\hline Candida albicans 12A & 0.020 & 0.049 \\
\hline Candida dubliniensis 7987 & -0.010 & 0.021 \\
\hline Candida dubliniensis 7988 & 0.034 & 0.049 \\
\hline
\end{tabular}

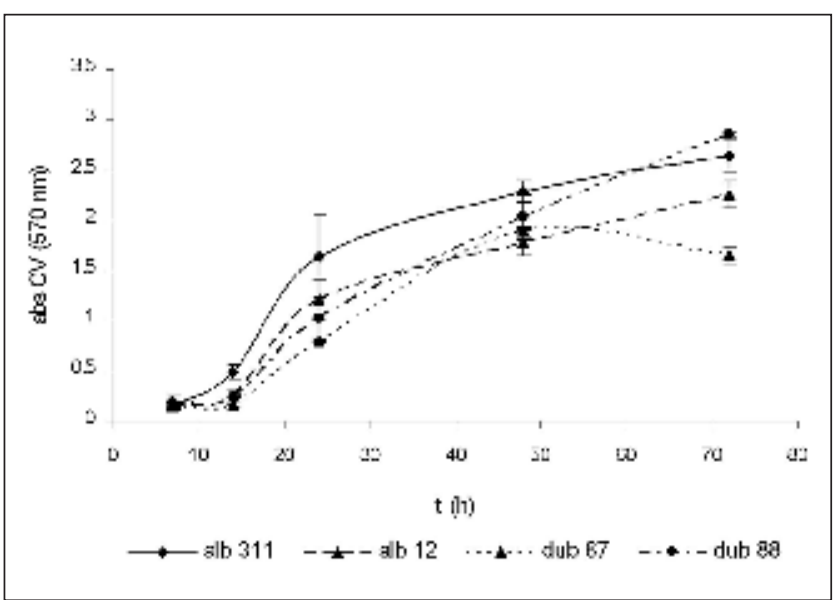

Fig. 2. Absorbance values of crystal violet solutions obtained from biofilms formed in SDB after 7, 14, 24, 48 and $72 \mathrm{~h}$ by C. albicans B311 (alb 311), C. albicans 12 A (alb12), C. dubliniensis 7987 (dub 87) and C. dubliniensis 7988 (dub 88).

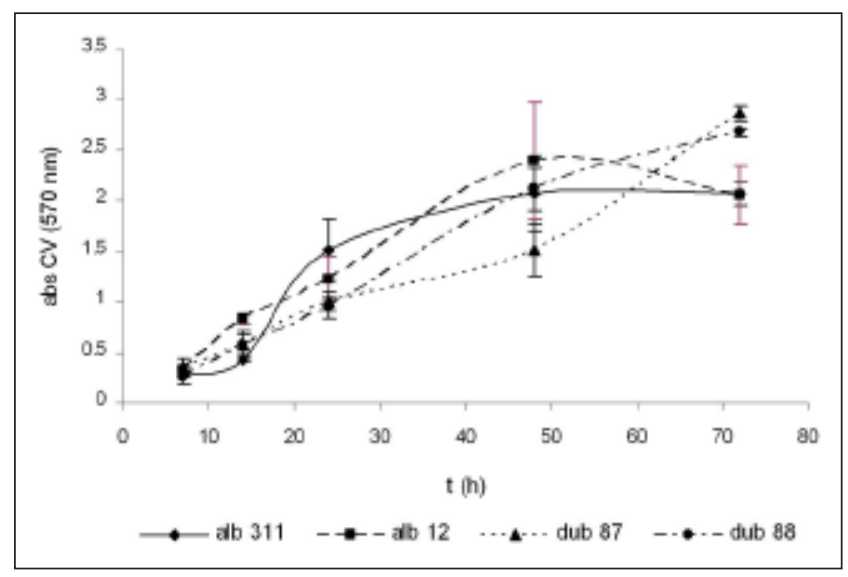

Fig. 3. Absorbance values of crystal violet solutions obtained from biofilms formed in artificial saliva after $7,14,24,48$ and $72 \mathrm{~h}$ by C. albicans B311 (alb 311), C. albicans 12 A (alb12), C dubliniensis 7987 (dub 87) and C. dubliniensis 7988 (dub 88).

basal cell layer may have an important role in anchoring the biofilm to the surface. ${ }^{24-26}$

Overall organisation of a candidal biofilm is generally similar to that of a bacterial biofilm, but details of its structure are highly dependent on the conditions under which it is formed. This plasticity in structure suggests that biofilms formed in the human host may also vary depending on the nature of the implanted device and in location. ${ }^{27}$ Variables such as surface material, ${ }^{28}$ medium, ${ }^{29}$ presence of other microorganisms, ${ }^{30}$ incubation conditions ${ }^{26}$ and cell density $^{31}$ can influence the structure of the resulting biofilm and the morphology of cells within it.

The demonstration that different conditions lead to different morphologies suggests that both morphological forms (yeast cells and hyphal cells) are capable of biofilm formation. To confirm this, Baillie and Douglas ${ }^{32}$ showed that a morphological mutant unable to produce hyphae formed a dense biofilm composed of blastopores, whereas a mutant that produced only filamentous cells formed a hyphal biofilm. 
One of the major conclusions of the present study is the fact that $C$. albicans and $C$. dubliniensis can form mature biofilms, whereas, for instances, Saccharomyces cerevisae can adhere but not form biofilm. ${ }^{26}$ This is important because it represents one of the pathogenic features of Candida species.

Another conclusion is that biofilm formation is either strain or species dependent. C. dubliniensis 7987 shows a different behaviour to that of other strain of the same species and the strains of C. albicans. Furthermore, C. albicans 12A and C. dubliniensis 7988 show similar behaviour in almost all conditions studied, with the exception of biofilm biomass formation in artificial saliva. C. albicans B311 also shows different behaviour from the other strains. Results on the biofilm activity of C. dubliniensis 7987 are similar to those obtained by Ramage et al. ${ }^{16}$

One of the goals of this work was the quantification of biofilm characteristics by two different methods (crystal violet and formation of tetrazolium salts [XTT]). The XTT

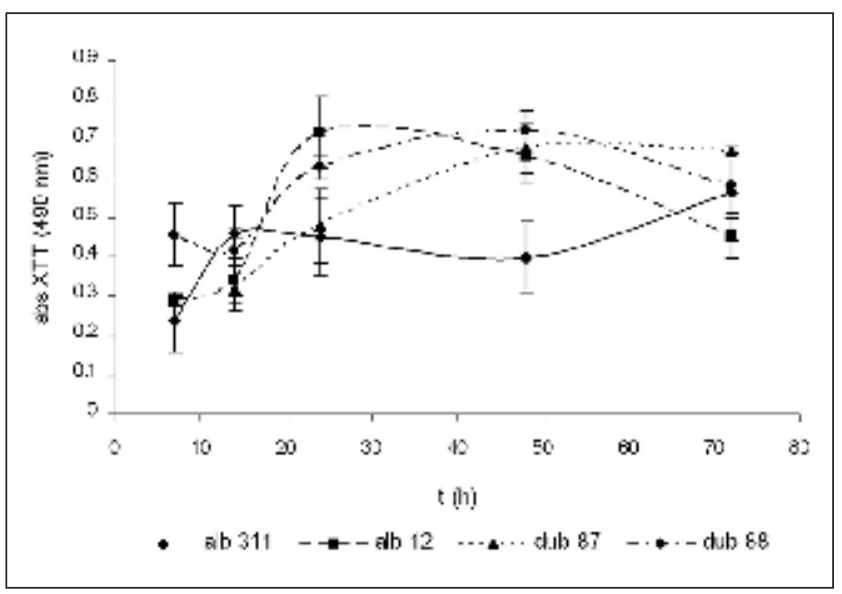

Fig. 4. Absorbance values of XTT solutions obtained from biofilms formed in SDB after 7, 14, 24, 48 and $72 \mathrm{~h}$ by C. albicans B311 (alb 311), C. albicans 12 A (alb 12), C. dubliniensis 7987 (dub 87) and C. dubliniensis 7988 (dub 88).

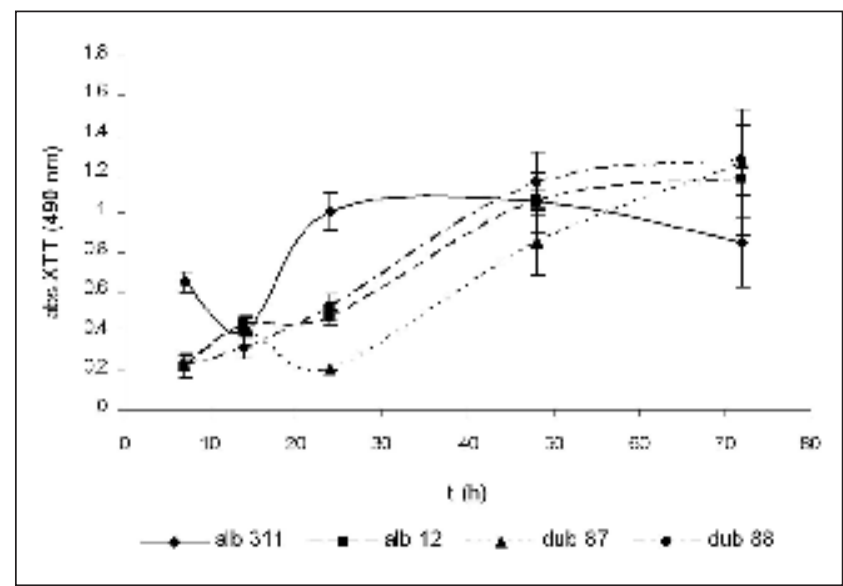

Fig. 5. Absorbance values of XTT solutions obtained from biofilms formed in artificial saliva after $7,14,24,48$ and $72 \mathrm{~h}$ by C. albicans B311 (alb 311), C. albicans 12 A (alb 12), C. dubliniensis 7987 (dub 87) and C. dubliniensis 7988 (dub 88).Fig. 2. Description of departmental results.
Table 3. Values of the slopes of the biofilm activity profiles (between 48 and 72 h) of C. albicans B311, C. albicans 12A, C. dubliniensis 7987 and C. dubliniensis 7988 evaluated with XTT, in both biofilm formation media.

\begin{tabular}{|c|c|c|}
\hline Strain & SDB & $\begin{array}{l}\text { Slope } \\
\begin{array}{l}\text { Artificial saliva growth } \\
\text { medium }\end{array}\end{array}$ \\
\hline Candida albicans B311 & 0.007 & -0.008 \\
\hline Candida albicans $12 \mathrm{~A}$ & -0.008 & 0.004 \\
\hline Candida dubliniensis 7987 & -0.000 & 0.016 \\
\hline Candida dubliniensis 7988 & -0.006 & 0.005 \\
\hline
\end{tabular}

method is usually used in studies on the susceptibility of Candida species biofilms to antifungal agents. ${ }^{33,34}$ However, Kuhn et al. ${ }^{35}$ described some limitations of this method. They conclude that it cannot be assumed that there is necessarily a linear relationship between the number of cells and the colorimetric signal, and that the relationship between XTT concentration and the resultant colorimetric signal is not necessarily proportional.

In the present study, the high correlation (Table 1) between absorbance of XTT and CV solutions means that these methods can be applied for cell quantification of suspended cultures at the exponential growth phase. Good correlation between the absorbance of CV and XTT solutions allows the use of both methods to compare differences in cell concentrations.

Yin et $a l .{ }^{36}$ reported that cell activity measured by XTT is associated linearly with the number of cells, confirming the reliability of XTT staining in biofilm quantification. However, as biofilm cells are enclosed in an exopolymeric matrix, this may limit access to nutrients and oxygen, resulting in possible alterations in cellular metabolic activity. If this is the case, the XTT assay, which is based on metabolic activity, may not determine accurately the number of cells. Other method, such as CV staining, should be used to quantify total biomass.

In comparing both methods of biofilm evaluation, it is interesting to note that biomass increases with time while activity decreases. This can be explained by the fact that a biofilm is composed of several cell layers and the basal ones may not be as active as those on the top of the biofilm.

Pseudohyphae formation is considered to be a virulence factor of Candida species and one of the determinants in adhesion interactions. ${ }^{37}$ The present results (Figs. 6 and 7) demonstrate that artificial saliva promotes the formation of pseudohyphae, but there was no evidence to suggest that pseudohyphae enhance biofilm formation.

Comparing both media, it is interesting to note that C. dubliniensis 7987 biofilm formation profiles show a positive and significant slope in artificial saliva, but the opposite happens in SDB. Table 4 shows the significant differences between the media are not constant, as they vary with evaluation method, strain and biofilm age.

Other studies involving the use of artificial saliva resulted in different conclusions. According to Jin et al. ${ }^{29}$ the presence of saliva does not significantly influence biofilm formation, while Nikawa et al. ${ }^{38}$ showed that the presence of saliva increases biofilm formation by C. albicans. 


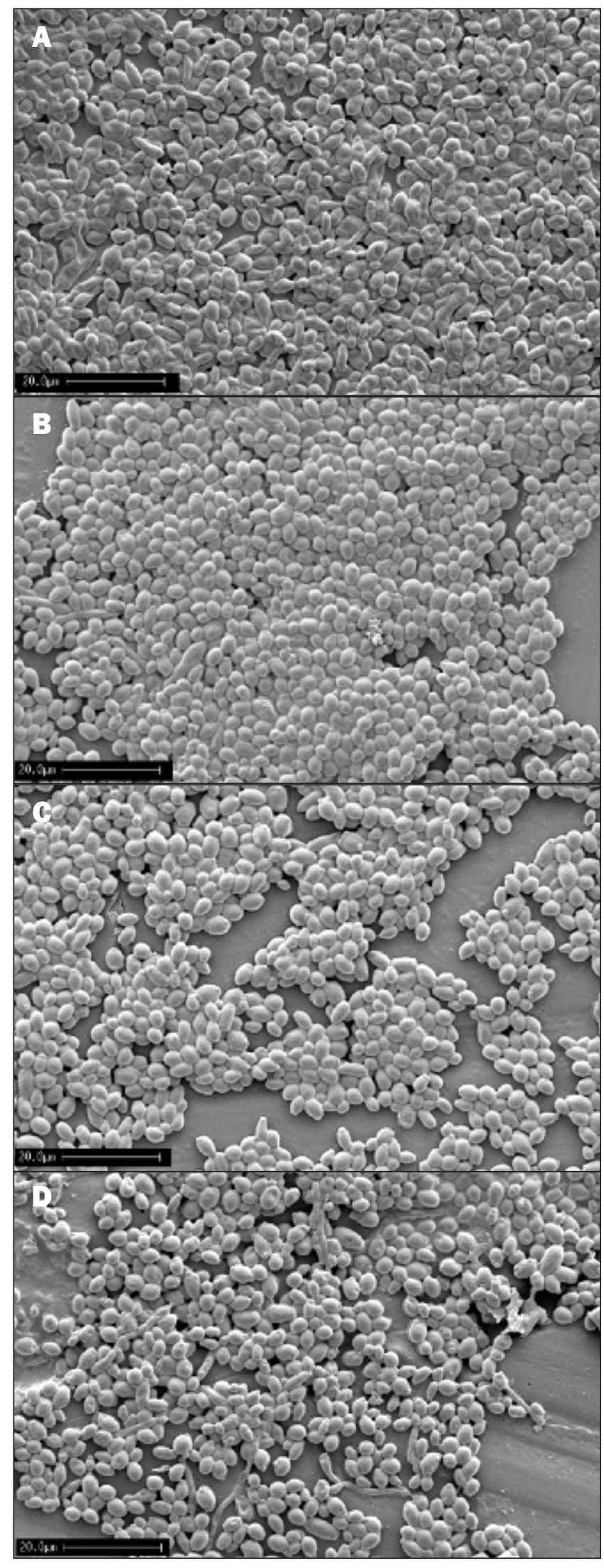

Fig. 6. SEM images of biofilms formed in SDB after $48 \mathrm{~h}$ for a) Candida albicans B311, b) C. albicans 12 A, c) C. dubliniensis 7987 and d) C. dubliniensis 7988 (original magnification $\times 1000$; bar represents $20 \mu \mathrm{m}$ ).

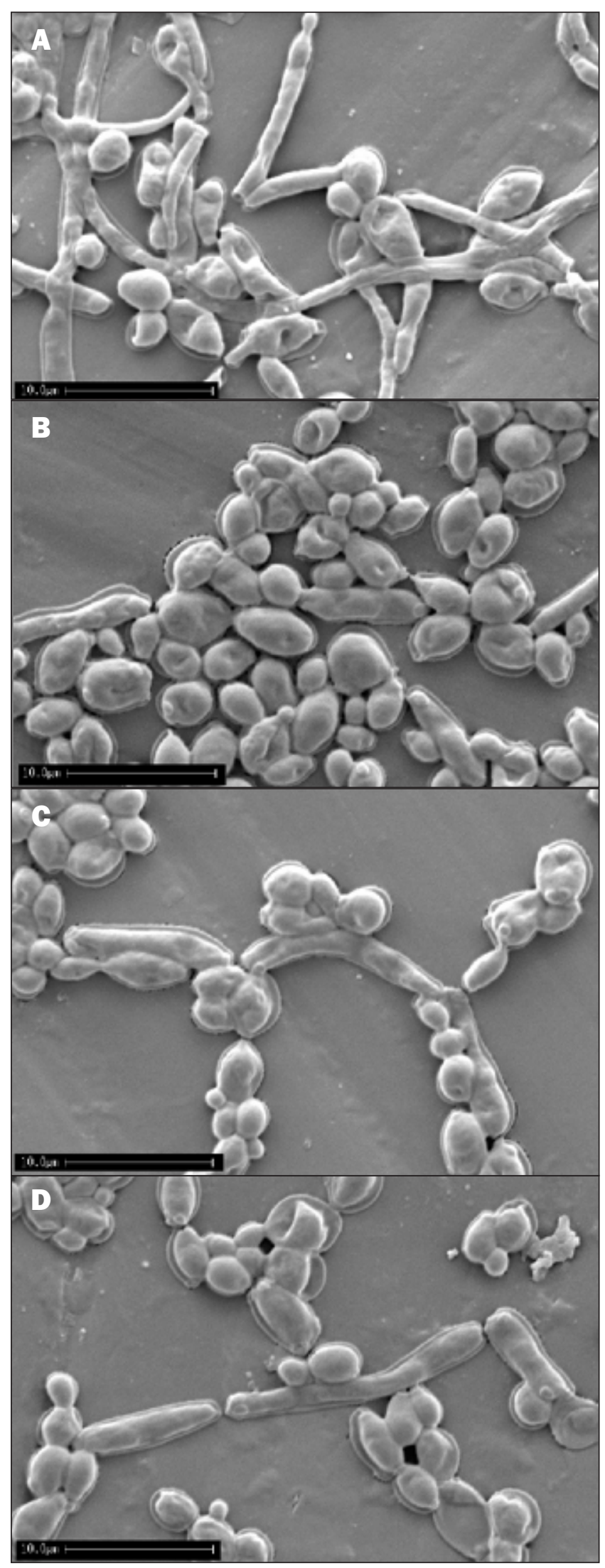

Fig. 7. SEM images of biofilms formed in artificial saliva after $48 \mathrm{~h}$ by a) Candida albicans B311, b) C. albicans $12 \mathrm{~A}$, c) C. dubliniensis 7987 and d) C. dubliniensis 7988 (original magnification x3000; bar represents $20 \mu \mathrm{m}$ ). 
Table 4. Significance values (P) obtained comparing biofilms formed by C. albicans B311 (alb 311), C. albicans 12 A (alb 12), C. dubliniensis 7987 (dub 87) and C. dubliniensis 7988 (dub 88) in SDB and artificial saliva growth medium, for both evaluation methods and all times assayed.

\begin{tabular}{|c|c|c|c|c|c|c|c|c|}
\hline \multirow[b]{3}{*}{ Time (h) } & \multirow[b]{3}{*}{ alb 311} & \multicolumn{6}{|c|}{ Significance value $(P)$} & \multirow[b]{3}{*}{ dub 88} \\
\hline & & \multicolumn{2}{|c|}{ CV } & \multicolumn{4}{|c|}{ XTT } & \\
\hline & & alb 12 & dub 87 & dub 88 & alb 311 & alb 12 & dub 87 & \\
\hline 7 & 0.005 & 0.009 & 0.041 & 0.382 & 0.009 & 0.142 & 0.002 & 0.016 \\
\hline 14 & 0.229 & 0.003 & 0.005 & 0.006 & 0.055 & 0.157 & 0.325 & 0.253 \\
\hline 24 & 0.683 & 0.922 & 0.460 & 0.571 & 0.002 & 0.038 & 0.020 & 0.137 \\
\hline 48 & 0.384 & 0.279 & 0.067 & 0.317 & 0.003 & 0.001 & 0.550 & 0.034 \\
\hline 72 & 0.007 & 0.305 & 0.026 & 0.481 & 0.122 & 0.000 & 0.001 & 0.003 \\
\hline
\end{tabular}

It is well known that some salivary proteins, such as human fibronectin and members of the proline-rich protein family, can act as receptors for C. albicans. On the one hand, these proteins, when immobilised on a surface, may promote candidal adhesion and subsequent biofilm formation by acting as receptors for free-living planktonic yeasts, while they may simultaneously block binding sites originally present on the substratum. In addition, the presence of salivary antimicrobial proteins has been demonstrated, ${ }^{39}$ which further complicates this issue. ${ }^{29}$

In the present study, the influence of saliva on biofilm formation was assessed using artificial saliva growth medium instead of natural saliva. Use of whole saliva has some disadvantages (donor and time of day variations) that can be overcome with an artificial solution.

Adhesion of strains used in the present study to acrylic was studied previously, ${ }^{19}$ and results showed that there were no statistical differences among them, either using water or artificial saliva. However, in terms of biofilm formation, $C$. dubliniensis 7987 showed different behaviour to that of $C$. albicans and C. dubliniensis 7988, both of which produced similar results. This difference was seen in biomass accumulation and cellular activity for both media (SBD and artificial saliva growth medium).

As a general conclusion, the number of initially adherent cells showed no direct correlation with biofilm evolution. Thus, adhesion and biofilm formation can be considered distinct factors of candidal virulence, and biofilm formation is strain dependent.

The authors fully acknowledge the financial support of FCT/Portugal through the grant BD3195/2000 and the project POCTI/BIO/42638/2001. We also would like to thank Dr. Célia Pais and Dr. Judite Almeida from the Department of Biology, University of Minho, for providing the Candida albicans 12A strain and Dr. Manuel Vilanova from Instituto de Ciêncicas Biomédicas Abel Salazar, University of Porto, for providing Candida albicans B311.

\section{References}

1 Sullivan DJ, Coleman DC. Candida dubliniensis: characteristics and identification. J Clin Microbiol 1998; 36: 329-34.

2 Sullivan DJ, Haynes K, Bille J et al. Widespread geographic distribution of oral Candida dubliniensis strains in human immunodeficiency virus-infected individuals. J Clin Microbiol 1997; 35: 960-4.
3 Jabra-Rizk MA, Falkler Jr WA, Merz WG, Baqui AAMA, Kelley JI, Meiller TF. Retrospective identification and characterization of Candida dubliniensis isolates among Candida albicans clinical laboratory isolates from human immunodeficiency virus (HIV)infected and non-HIV-infected individuals. J Clin Microbiol 2000; 38: 2423-6.

4 Calderone RA. Candida and Candidiasis. Washington DC: ASM Press, 2002.

5 Donlan RM, Costerton JW. Biofilms: survival mechanisms of clinically relevant microorganisms. Clin Microbiol Rev 2002; 15: 167-93.

6 Mukherjee PK, Chandra J. Candida biofilm resistance. Drug Resist Update 2004; 7: 301-9.

7 Donlan RM. Biofilms and device-associated infections. Emerg Infect Dis 2001; 7: 277-81.

8 Costerton JW, Stewart PS, Greenberg EP. Bacterial biofilms: a common cause of persistent infections. Science 1999; 284: 1318-22.

9 Jenkinson HF, Douglas LJ. Interactions between Candida species and bacteria in mixed infections. In: Brogden KA, Guthmiller JM eds. Polymicrobial diseases. 2002: 357-73.

10 Donlan RM. Biofilms: microbial life on surfaces. Emerg Infect Dis 2002; 8: 1-9.

11 Lewis K. Riddle of biofilm resistance. Antimicrob Agents Chemother 2001; 45: 999-1007.

12 Bachmann SP, Walle KV, Ramage $\mathrm{G}$ et al. In vitro activity of caspofungin against Candida albicans biofilms. Antimicrob Agents Chemother 2002; 46: 3591-6.

13 Baillie GS, Douglas J. Matrix polymers of Candida biofilms and their possible role in biofilm resistance to antifungal agents. $J$ Antimicrob Chemother 2000; 46: 397-403.

14 Kuhn DM, Chandra J, Mukherjee PK, Ghannoum MA. Antifungal susceptibility of Candida biofilms: unique efficacy of amphotericin B lipid formulations and echinocandins. Antimicrob Agents Chemother 2002; 46: 1773-80.

15 Hawser SP, Baillie GS, Douglas J. Production of extracelular matrix by Candida albicans biofilms. J Med Microbiol 1997; 47: 253-6.

16 Ramage G, Walle KV, López-Ribot J. Biofilm formation by Candida dubliniensis. J Clin Microbiol 2001; 39: 3234-40.

17 Jabra-Rizk MA, Falkler WA, Meiller TF. Fungal biofilms and drug resistance. Emerg Infect Dis 2004; 10: 14-9.

18 Kirkpatrick WR, López-Ribot JL, Mcatee RK, Patterson TF. Growth competition between Candida dubliniensis and Candida albicans under broth and biofilm growing conditions. J Clin Microbiol 2000; 38: 902-4.

19 Henriques M, Azeredo J, Oliveira R. Adhesion of Candida albicans and Candida dubliniensis to acrylic and hydroxipapatite. Coll Surf B: Biointer 2004; 33: 235-41. 
20 Gal J, Fovet Y, Adib-Yadzi M. About a synthetic saliva for in vitro studies. Talanta 2001; 53: 1103-15.

21 Johnson LE, Meyers NL, Jackson GA. Inhibition of the growth of Streptococcus mutans to a glass surface. 147th Ordinary SGM Meeting 2000; 10.

22 Cotter G, Kavanagh K. Adherence mechanisms of Candida albicans. Br J Biomed Sci 2000; 57: 241-9.

23 Samaranayke LP, MacFarlane TW. An in vitro study of the adherence of Candida albicans to acrylic surfaces. Arch Oral Biol 1980; 25: 603-9.

24 Douglas LJ. Medical importance of biofilms in Candida infections. Rev Iber Micol 2002; 19: 139-43.

25 Douglas J. Candida biofilms and their role in infection. Trend Microbiol 2003; 11: 30-6.

26 Chandra J, Kuhn DM, Mukherjee PK, Hoyer LL, McCormick T, Ghannoum MA. Biofilm formation by the fungal pathogen Candida albicans: development, architecture and drug resistance. J Bacteriol 2001; 183: 5385-94.

27 Kumamoto CA. Candida biofilms. Curr Opin Microbiol 2002; 5: 608-11.

28 Hawser SP, Douglas LJ. Biofilm formation by Candida species on the surface of catheter materials in vitro. Infect Immun 1994; 62: 915-21.

29 Jin Y, Samaranayke LP, Samaranayke YH, Yip HK. Biofilm formation of Candida albicans is variably affected by saliva and dietary sugars. Arch Oral Biol 2004; 49: 789-98.

30 Adam B, Baillie GS, Douglas J. Mixed species biofilms of Candida albicans and Staphylococcus epidermidis. J Med Microbiol 2002; 51: 344-9.

31 Hornby JM, Jensen EC, Lisec AD et al. Quorum sensing in the dimorphic fungus Candida albicans is mediated by farnesol. Appl Environ Microbiol 2001; 67: 2982-92.

32 Baillie GS, Douglas LJ. Role of dimorphism in the development of Candida albicans biofilms. J Med Microbiol 1999; 48: 671-9.
33 Meletiadis J, Mouton JW, Meis JFG et al. Comparison of spectrophotometric and visual readings of NCCLS method and evaluation of a colorimetric method based on reduction of soluble tetrazolium salt, 2,3-bis\{2methoxy-4-nitro-5-[(sulfenylamino) carbonyl]-2Htetrazolium-hydroxide\}, for antifungal susceptibility testing of Aspergillus species. J Clin Microbiol 2001; 39: 4256-63.

34 Hawser SP, Norris H, Jessup CJ, Ghannoum MA. Comparison $2,3$ - bi s ( 2 - m e th ox y - 4-nit ro - 5 - s ulf e n y 1$)-5$ [(Phenylamino)carbonyl]-2H-tetrazolium hydroxide (XTT) colorimetric method with the standardized national committee for clinical laboratory standards method of testing clinical yeast isolates for susceptibility to antifungal agents. J Clin Microbiol 1998; 36: 1450-2.

35 Kuhn DM, Balkis M, Chandra J, Mukherjee PK, Ghannoum MA. Uses and limitations of the XTT assay in studies of Candida growth and metabolism. J Clin Microbiol 2003; 41: $506-8$.

36 Jin Y, Yip HK, Samaranayke YH, Yau JY, Samaranayke LP. Biofilm forming ability of Candida albicans is unlikely to contribute to high oral yeast carriage in human immunodeficiency virus infection. J Clin Microbiol 2003; 41: 2961-7.

37 Brown AJP. Expression of growth form-specific factors during morphogenesis in Candida albicans. In: Calderone RA ed. Candida and candidiasis. Washington DC: ASM Press, 2002: 87-93.

38 Nikawa H, Nishimura H, Hamada T, Kumagai H, Samaranayke L.P. Effects of dietary sugars and, saliva and serum on Candida biofilm formation on acrylic surfaces. Mycopathologia 1997; 139: 87-91.

39 San Millan R, Elguezabal N, Regulez P, Moragues MD, Quindos G, Ponton J. Effect of salivary secretory IgA on the adhesion of Candida albicans to polysterene. Microbiology 2000; 146: 2105-12. 